\begin{tabular}{c|c} 
EESD'15 & The $^{\text {th }}$ International Conference on Engineering Education for Sustainable Development \\
\cline { 2 - 2 } & Vancouver, Canada, June 9 to 12, 2015
\end{tabular}

\title{
DEVELOPING GLOBAL PREPAREDNESS EFFICACY
}

\author{
Bhavna Hariharan ${ }^{1,2}$ and Sneha Ayyagari ${ }^{1}$ \\ ${ }^{1}$ Stanford University, USA \\ 2 bhavnah@stanford.edu
}

\begin{abstract}
Increasing globalization and technological innovations have redefined the role of engineers in working towards sustainable development. The question of how to measure and evaluate preparedness of engineering students to meet these requirements remains an open question. This paper develops a performance indicator called Global Preparedness Efficacy (GPE) to measure the effectiveness of curricula that bring student engineers together with underserved communities in satisfying ABET criteria $3 \mathrm{~h}$, which is "the broad education necessary to understand the impact of engineering solutions in a global, economic, environmental, and societal context". This indicator measures ability to navigate the complexity and novelty of the problem space and enabling the creation solutions to the problem at hand consistent with the global socio-economic, political and cultural realities.
\end{abstract}

\section{INTRODUCTION}

Increasing globalization and technological innovations have redefined the role of engineers in working towards sustainable development. This is reflected in the creation and adoption of ABET Engineering Criteria 2000 which included six professional skills to prepare engineers who were more aware of how their profession, products and services are embedded in the larger global, socio-economic and political context (Shuman et al 2005). The question of how to measure and evaluate preparedness of engineering students to meet these requirements remains an open question. This paper describes a theoretically derived metric global preparedness efficacy as one method to evaluate the effectiveness of a curriculum.

Responding to the need to prepare student engineers to collaborate with underserved communities, the Global Engineer's Education (GEE) program was implemented at Stanford University. The GEE curriculum offers students the opportunity to work with rather than for a community in rural India by understanding the problem space, practicing methods of ethical collaboration, and prototyping technologies to address sanitation and hygiene issues in the area. The curriculum fosters this collaboration through three unique curricular elements. Regular video calls with experts at the partner organization in India, the Environmental Sanitation Institute (ESI) allowed students to engage directly with community members. Readings and discussions from various disciplines encouraged students to consider the complexity of the problem space of sanitation and hygiene as they prototyped technologies. The course focused on the idea of designing with care by inviting students and community members to express their values and goals and incorporate each of these "care statements" into the final design.

These three curricular aspects allow students to engage with the community in a way that positions student engineers to incorporate their own values and ideas in context of local realities and knowledge. This approach is consistent with the work of Sheri Sheppard et al who state in their book Educating Engineers: Designing for the Future of the Field that, "The shift from an outside to an inside perspective can be understood as a shift from engineering for "them" to engineering for "us". Although this new point of view may be disarming, at the same time it holds the potential to inspire new thinking, for a shift from 
an outside to an inside perspective highlights the complex social, physical and informational interconnections" (Sheppard et al 2009).

The GEE course curriculum provides the opportunity for students to experience the challenges in collaborating with an underserved community globally and conceiving solutions to the challenges faced by the community that is mindful of and responds to the local economic, environmental, social, political, ethical and cultural conditions in a way that is safe. As such, students have the opportunity to examine subjectively daunting concerns they may have about bridging language and cultural barriers and connecting with the harsh realities that the underserved communities experience in a non-threatening environment. Knowing that their communities are real and the regular real-time connection with them also imposes an ethical responsibility on the students allowing them direct experience of real work conditions. As such, GEE serves as a good case to determine global preparedness efficacy of student engineers.

\section{A THEORETICAL UNDERSTANDING OF GLOBAL PREPAREDNESS EFFICACY (GPE)}

The seminal work of John Dewey was selected above all the others because of his trans-actional worldview and his particular emphasis on learning through doing. He also explicitly focuses on learning as a result of interplay between the individual and the environment.

For Dewey, learning is a social process. It happens as a result of individuals interacting with the larger social environment in which they are situated. In fact, he went as far as to claim that education was a means of social continuity. Individuals learned from society and gave back to it, new knowledge that they discovered. Dewey believes it is an undeniable fact that all individuals grow up in a social medium.

Therefore, understanding learning by isolating the individual from the environment is fallacious: "As a matter of fact every individual has grown up, and always must grow up, in a social medium. His responses grow intelligent, or gain meaning, simply because he lives and acts in a medium of accepted meanings and values through social intercourse, through sharing in the activities embodying beliefs, he gradually acquires a mind of his own. The conception of mind as a purely isolated possession of the self is at the very antipodes of the truth... the self is not a separate mind building up knowledge anew of its own account" (Dewey 1969-1990).

\subsection{Dewey's Trans-Action Model, Experience and Learning}

His views on learning and education stem from his trans-action worldview. It is a perspective "where systems of description and naming are employed to deal with aspects and phases of action, without final attribution to 'elements' or other presumptively detachable or independent 'entities', 'essences', or 'realities', and without isolation of presumptively detachable 'relations' from such detachable 'elements'" (Dewey and Bentley 1949). A trans-actional perspective considers a system holistically without attributing intention or will to individual entities. Entities derive their meaning from the context in which they are embedded. They are neither given predefined wills or intentions, nor are they looked at in isolation from the context in which they are embedded.

In the case of GEE, students considered the complexities of the sanitation and hygiene space from a holistic perspective by analyzing multidisciplinary views on the topic. The course focused on the broader perspective of designing with care and working with communities, allowing students a perspective through which they could approach and overcome discontinuity events.

A discussion of Dewey's idea of learning begins with understanding his concept of Experience. Experience for Dewey is an active-passive process. It has an active component where individuals act on their environment. Individuals experience the consequences of their actions in the complementary passive phase. He describes that learning occurs "[w]hen we experience something we act upon it, we do something with it; then we suffer or undergo the consequences. We do something to the thing and then it does something to us in return: such is the peculiar combination [of experience]" (Dewey 1969-1990, p. 163). 
Learning is the creation of continuities and relationships between actions and their results and consequences. To learn from experiences, it is necessary for the individual to "continue into the undergoing of consequences, when the change made by action is reflected back into a change made in us, the mere flux is loaded with significance, we learn something." By moving back and forth between actions and the undergoing, it is possible to learn about the connections between actions and their effects: "Under such conditions, doing becomes trying; an experiment with the world to find out what it is like; the undergoing becomes instruction - discovery of the connection of things" (Dewey 1969-1990, p. 164). According to Dewey, a learning experience has two complementary components, namely active doing and undergoing. Consistent with the trans-actional point of view, learning is a combined experience of an individual actively acting on the environment (whereby the individual can alter and change the context or respond to a stimulus or problems in a given environment) while simultaneously the environment acts upon the individual.

When working with underserved communities, the students, researchers, and instructors often encounter completely novel contexts. Initiatives that bring together students and underserved communities are usually trying to bridge social, economic, linguistic, political, geographic and cultural differences. As such it is very common that the undergoing is unexpected. In fact, in an NSF sponsored study, a book published by Gary Downey captures the experience of a Professor at University of Denver at Colorado who decided not to pursue international engineering work because she felt "depressed to know that the large body of knowledge in the development area was not readily accessible or available to engineers either in their curricula or their international practice." She acknowledged that the disconnect between the values and experiences of community agendas, NGO partners, and student engineers made it difficult to "honestly assess their own practices and their unintended consequences."(Downey and Beddoes 2011, pg. 162)

\subsection{Applying Zimbardo's Discontinuity Theory}

An explanation for these aberrant experiences of undergoing can be found in Philip Zimbardo's Discontinuity Theory. It investigates and proposes a model of how individuals adapt to aberrant experiences in their lives. He defines discontinuity as "a violation of the expectation in any domain of functioning highly valued self" (Zimbardo 1999, p. 345). It is an event that proves to be disruptive to the normal flow and pattern of everyday life.

Experiencing a discontinuity "involves an awareness of a noticeable deviation from an expected normative standard of how one usually feels, thinks, perceives, or acts - in those areas that figure into the calculation of one's global self" (Zimbardo 1999, p. 351). It implies that the discontinuities are felt in cases in which a highly valued self-image of the individual is threatened. It is only the disruption of patterns to which an individual is deeply attached, or holds in high regard as an essential character trait, that creates experiences of discontinuity. The nature of discontinuities is such that they are disruptive enough to cause an individual to seek an explanation for the experience in an attempt to restore either the previously experienced sense of normalcy or a renewed sense of self.

Zimbardo's theory offers nine types of "violations of expectations" (V.O.E) as sources of discontinuity, "each of which typically elicits characteristic affective reactions" (Zimbardo 1999, p. 351). The V.O.E are misfortune, good fortune, magic, miracle, humor, horror, natural disasters/cosmic perturbations, social deviance, aesthetic value violation (Zimbardo 1999, p. 351-352). It is reasonable to expect that any student working in a cross-cultural context in the developing world will encounter such violation of expectations. It is natural to imagine that differences in culture, economic status, belief structures and personal histories will lead to discontinuities.

According to Zimbardo's discontinuity theory, discontinuities can lead to inquiries that lead to new understandings, but they may also result in confused or biased behavior that can have less desirable outcomes. The experience of discontinuity is one of uncertainty, in which confusion about the expected behavior of learning and teaching results in the inability to proceed with the project. The discontinuity is overcome when a new course of action or renewed action is restored. From a Deweyan perspective, 
discontinuities are experiences of aberrant undergoing where active doing is lost and can be potential learning experiences if the active doing is restored.

\section{DEFINING GLOBAL PREPAREDNESS EFFICACY}

Using the Dewey-Zimbardo framework described above, it is possible to look at student experiences when participating in project-based course that enable engagement with underserved communities. The metric is being developed with an aim to measure the ability of students to navigate the complexity and novelty of the problem space and enabling the creation solutions to the problem at hand consistent with the global socio-economic, political and cultural realities. Identifying the number of discontinuities encountered and identifying whether or not they were converted to learning experiences (as indicated by renewed action) could serve as a good measure for global preparedness. Global Preparedness Efficacy is defined as the ratio of resolved to total discontinuity events. This would imply that the closer the ratio to 1 , the better the students were prepared for global engagement.

\subsection{Calculating GPE}

To calculate GPE, a necessary step is to determine whether discontinuities could be coded from student journals. A coding scheme to identify discontinuities and renewed action was developed using the method shown in Figure 1. This meta-framework for generating a coding scheme (MFCS) was developed by studying two emotion-coding schemes: Intimacy Coding Scheme and the Specific Affect Coding System (SPAFF) (Hariharan 2011).

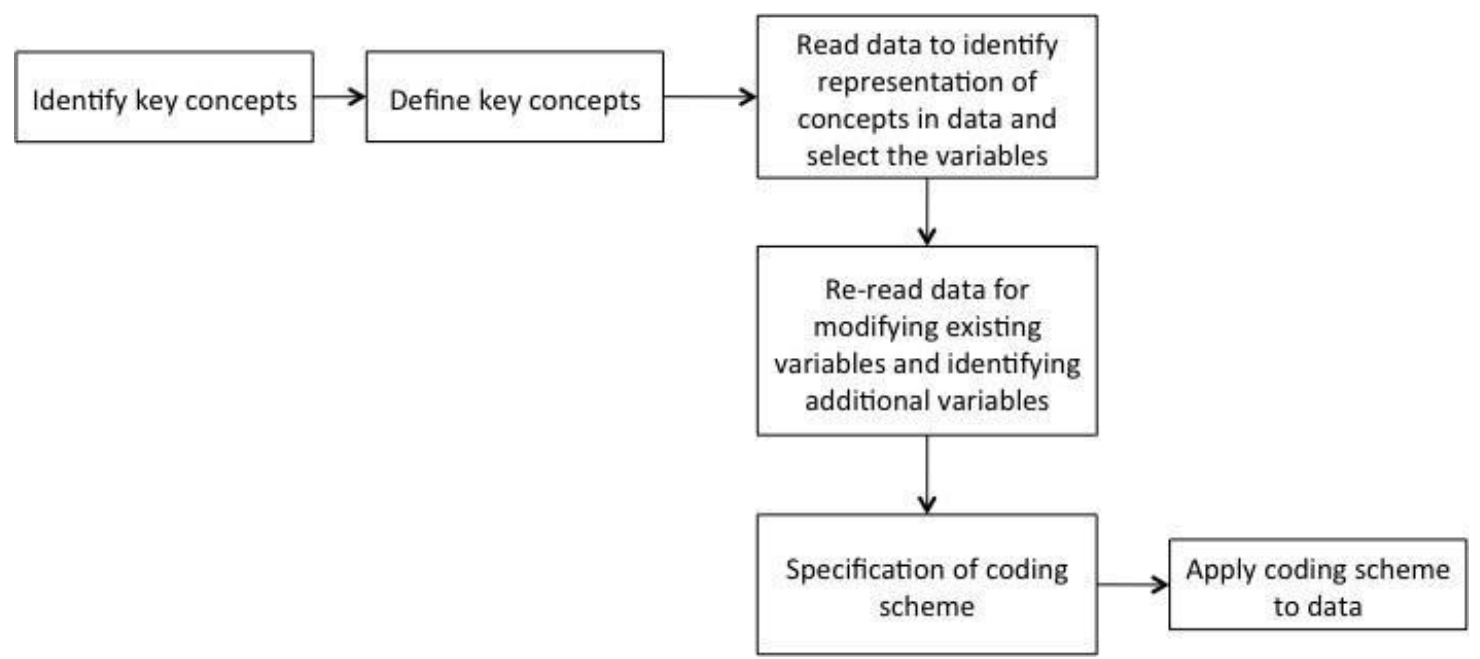

Figure 1: Meta Framework for generating a Coding Scheme (MFCS)

The first step in the process, as shown in Figure 1 was to recognize the key concepts from the theory underlying the interactions of interest. Dewey's Learning Theory and Zimbardo's Discontinuity Theory were used to delineate the transition from discontinuity to learning experiences. As described previously, experiences of discontinuity are those where active doing stops and a shift to learning experience happens when active doing is restored or there is engagement in renewed action. Thus discontinuity and renewed action emerged as the key concepts. They were defined as follows:

- Discontinuity: Drawing from Zimbardo's theory, discontinuity is experienced when a highly valued expectation/belief is violated. In the case of the student practitioners, these discontinuities are likely to come from their assumptions about the community practitioners and their expertise in engineering design.

- Renewed Action: Action that comes after a discontinuity has been understood and overcome has been called renewed action. The naming is deliberate to differentiate it from merely resuming the same 
action that was being under- taken prior to experiencing a discontinuity. Renewed action implies acting out of having understood and contextualized the discontinuity.

- Vulnerability: Expressions of emotions, reflections and at times statements made in the third person about the topic under discussion. These were representative of the student practitioners' transition from discontinuity to renewed action. The feeling of exposure resulted in these expressions being collectively called "vulnerability".

The next stage was reading the data to identify how discontinuity and renewed action in the vulnerability were represented in the data.

\subsubsection{Identifying Discontinuities}

On reading through the data, it became apparent that discontinuities were at times explicitly expressed. In other cases, they were implied in expressions of struggle and in a few cases expressions of joy. The most obvious representation of discontinuity was as an expression of surprise or concern as shown in the following example:

We also discussed our reactions to the assigned readings for the week. All of us were very taken back by the readings and had never thought of safety as an issue linked to toilets (Student 614).

Discontinuities were not always explicitly expressed. They were also implied in texts that spoke of the struggle or joy that the student practitioner had experienced. In such cases, the discontinuity was coded as the event upon which the student practitioner was reflecting on. For example, one student wrote Even though we met for 2 hours, we did surprisingly little (Student 514). This statement in itself was not the discontinuity. The students were prototyping a bike-powered fan to improve ventilation in the toilet space.

\subsubsection{Identifying Renewed Action}

Following identification of discontinuities in the data, evidence for renewed action was sought. These were statements that expressed the student practitioners' understanding of the discontinuity and their intention to re-engage in active doing. For example, the two instances of discontinuities presented in the previous section were associated with the following renewed action:

Discontinuity: We also discussed our reactions to the assigned readings for the week. All of us were very taken back by the readings and had never thought of safety as an issue linked to toilets.

Renewed Action: If we can create a toilet that is good enough to have very close to the school, so students don't have to venture too far to use the bathroom. Also, making sure people, not only are, but feel safe, is something important to me. Because we are most influenced by our earliest experiences, having things tailored to children and young girls especially would be something that I'm interested in.

Discontinuity: Even though we met for 2 hours, we did surprisingly little.

Renewed Action: However, we realized some key aspects - we needed to buy a chain delinker, and we needed to build a stand for the bike so that one could pedal in a solitary position.

In both instances of discontinuities above, we see that a discontinuity has been understood and there is an indication of how to move forward. However, not all discontinuities were, associated with renewed action. Discontinuities that had associated renewed action were called "resolved discontinuities" while those that did not have renewed action were called "unresolved" discontinuities. Since representations of both key concepts were found in the data without modification of concepts, discontinuity and renewed action were chosen as the variables of the coding scheme.

The instances of vulnerability associated with the examples of discontinuities and renewed action presented above shown below. 
Discontinuity: We also discussed our reactions to the assigned readings for the week. All of us were very taken back by the readings and had never thought of safety as an issue linked to toilets.

Vulnerability: This was an issue discussed in the context of sub-Saharan Africa, but we are all curious as to whether or not this applies to the village we are working with as well.

Renewed Action: If we can create a toilet that is good enough to have very close to the school, so students don't have to venture too far to use the bathroom. Also, making sure people, not only are, but feel safe, is something important to me. Because we are most influenced by our earliest experiences, having things tailored to children and young girls especially would be something that I'm interested in.

Discontinuity: Even though we met for 2 hours, we did surprisingly little.

Vulnerability: We were crippled by a lack of adequate tools, as well as inexperience with disassembling bike parts. All we managed to get done, in terms of prototyping, was separate the rear-wheel of a bike. It sounds really minute, now that I think of it, but the process was a lot more complicated.

Renewed Action: However, we realized some key aspects - we needed to buy a chain delinker, and we needed to build a stand for the bike so that one could pedal in a solitary position.

A second reader independently applied the coding scheme to verify that the three variables (discontinuity, vulnerability, and renewed action) were clearly defined and properly represented the data. The collective of discontinuity and associated vulnerability and renewed action (if it existed) were labeled as a discontinuity event. The coded data were put into tables as shown in Table 1 to enable easy retrieval for future use.

Table 1: Example of Coding Scheme

\begin{tabular}{ll}
\hline Discontinuity & $\begin{array}{l}\text { I was very taken back by the Geographies of Danger paper, in } \\
\text { which issues of girls specifically facing the danger of rape when } \\
\text { going to the bathroom in sub-Saharan Africa. }\end{array}$ \\
\hline Vulnerability & $\begin{array}{l}\text { This was extremely alarming for me, as safety has never been an } \\
\text { issue that I would consider when going to the bathroom. }\end{array}$ \\
\hline \multirow{2}{*}{ Renewed } & $\begin{array}{l}\text { Several of the girls noted that in female bathrooms, girls often } \\
\text { socialize, but in dorms where there are "gender-neutral" } \\
\text { bathrooms, this socializing does not necessarily happen. For our } \\
\text { toilet design, it might be interesting to think about how to cater the } \\
\text { toilet to each specific gender using it so that both are } \\
\text { comfortable. }\end{array}$ \\
\hline
\end{tabular}

A total of 334 journal entries from 16 students of the GEE class were coded. A total of 130 discontinuity events were found, off which, 107 were resolved. The Global Preparedness Efficacy for GEE, was found to be 0.82 .

Table 2: Summary of data

Summary of 2014 data

\begin{tabular}{lc}
\hline Discontinuities & 104 \\
\hline Resolved & 85 \\
\hline Unresolved & 19 \\
\hline \multicolumn{2}{c}{ Summary of 2013 data } \\
\hline
\end{tabular}




\begin{tabular}{ll}
\hline Discontinuities & 26 \\
\hline Resolved & 22 \\
\hline Unresolved & 4 \\
\hline & Total data \\
\hline Discontinuities & 130 \\
\hline Resolved & 107 \\
\hline Unresolved & 23 \\
\hline
\end{tabular}

\section{DISCUSSION}

The presence of 130 discontinuity events shows that the GEE course made it possible for the students to experience the reality of working across cultural, social, economic, linguistic and geographical differences. GEE is a good setting to measure GPE because the curriculum allows students to experience the hurdles that they would encounter in field settings. The high number of resolved discontinuity events (107) indicates that GEE was also able to make what could potentially be an overwhelming experience of aberrant undergoing into a learning environment.

The difference in number of discontinuities observed in 2013 and 2014 can be attributed to two main reasons. The number of students in the class in 2013 (when the course was first offered) was 8 while in 2014 the class size was 11. The total number of journal entries written in the 2013 offering of the class was 65 while in 2014 the number was 269. This discrepancy is attributable to the fact that in 2013 the course was offered as credit/no credit while in 2014 the course was offered for letter grade with journal entries contributing to $25 \%$ of the total grade.

When looking at the data for individual students for 2014, there was a wide variation in number of discontinuities encountered. The range of the data was 17 (2 and 19 being the highest and lowest number recorded). A possible explanation for this large range is that there was no given format or structure for journaling. The students were simply asked to take note of their activities with regard to the course and reflect on it. This is an avenue for improvement and questions about providing prompts for the journals is being discussed and researched.

\section{IMPLICATIONS AND FUTURE WORK}

The primary purpose of this research was to describe the development of GPE as a metric to evaluate global engineering curricula. In addition, the paper describes a coding scheme used to determine GPE and how it was used with data collected in the GEE course. The data showed that GEE had a high GPE. Further research is required to study what aspects of the GEE course contribute to satisfying ABET criteria $3 \mathrm{~h}$ that is the broad education necessary to understand the impact of engineering solutions in a global, economic, environmental, and societal context (Shuman et al 2005).

Studying the causes of discontinuities across the data set can assist in making curricular innovations. Another application of the coding scheme is to monitor student experience and progress throughout the course. Regular coding will allow instructors to monitor what causes individual students to encounter discontinuities. 


\section{Acknowledgements}

The authors would like to thank the scholars at the Kozmetsky Global Collaboratory, field collaborators at the Environmental Sanitation Institute in India, and all of the students of the Global Engineers' Education course for their support in conducting this research. The authors extend a special thank you to Professor Sheri Sheppard and Dr. Syed Shariq who helped clarify and name global preparedness efficacy.

\section{References}

Dewey, J. 1969-1990. The Collected Works of John Dewey: The Early Works 1882-1898 [5 vols.], The Middle Works 1899-1924 [15 vols.] and The Later Works 1925-1952 [17 vols.]. Carbondale: Southern Illinois University Press, USA.

Dewey, J. Bentley, A F. 1949. Knowing and the Known. Boston: Beacon Press, USA.

Downey, G. Beddoes, K. 2011. What is Global Engineering Education For? The Making of International Educators - Parts I and II. Morgan and Claypool, USA

Hariharan, B. 2011. Innovating capability for continuity of inquiry in the face of discontinuity within the context of engineering education research. PhD Dissertation, Stanford University, USA

Sheppard, S S. Macatangay, K. Colby, A. Sullivan, W. 2009. Educating Engineers: Designing for the Future of the Field. Jossey-Bass, A Wiley Imprint USA.

Shuman, L J. Besterfield-Sacre, M, \& McGourty, J. 2005. The ABET "professional skills"—can they be taught? Can they be assessed?. Journal of Engineering Education, 94(1), 41-55.

Zimbardo, P. 1999. Discontinuity Theory: Cognitive and Social Searches for Rationality and Normality May lead to Madness. Chapter: Advances in Experimental Social Psychology, Vol 31. Academic Press, USA. 